\title{
Relations Power Characteristics of High-Speed Chucks with Power Cuts
}

\author{
FW El-Dahabi \\ Department of Mechanic, Faculty of Engineering, Lebanese University, Tripoli, Lebanon.
}

Correspondence Author: FW El-Dahabi, Department of Mechanic, Faculty of Engineering, Lebanese University, Tripoli, Lebanon.

E-mail: -dahabi@i.ua

Received date: 20 May 2019, Accepted date: 20 July 2019, Online date: 28 July 2019

Copyright: (ㅇ 2019 FW El-Dahabi $e t$ al, This is an open-access article distributed under the terms of the Creative Commons Attribution License, which permits unrestricted use, distribution, and reproduction in any medium, provided the original author and source are credited.

\begin{abstract}
The effect of cutting forces on the clamping force in high-speed chucks been analyzed at different rotational speeds of the spindle, these speeds determine the magnitude of the centrifugal forces of the unbalanced chucks. The above consideration, design schemes and analytical dependencies can be used to desigh high-speed clamping chucks under given cutting conditions.
\end{abstract}

Keywords: high-speed chuck, centrifugal force, cutting force, gain, spindle

\section{INTRODUCTION}

Existing progressive cutting tools allow a high cutting speed. Therefore, to increase the processing capacity, modern CNC lathes provide high spindle speeds (up to 20,000 rpm) (Al - Quraan,2016; Kuznetsov et al.,1988). However, as the machine spindle speed increases, the action of the centrifugal forces on the clamping chuck (CC) cams increases significantly, reducing the clamping force, limiting the cutting regimes, which reduces the processing capacity and reduces the possibility of maximum utilization of the machine's power. This circumstance requires the creation of new designs of high-speed clamping chucks (HSCC) capable of operating at high speeds of the spindle and retaining the clamping force at high cutting conditions (Kuznetsov et al.,2010). In some cases, the calculation of clamping forces has a verification nature. The required clamping force, calculated from the processing conditions, must be less than or equal to the force developed by the clamping device being used. If this condition is not met, then the processing mode is changed to reduce the necessary clamping force and perform a verification calculation. The solution of the problem can be carried out in the reverse order. Then, according to the known clamping force of the available device, the permissible cutting forces are determined, and the processing conditions are set. The main objective of this study so establishes the effect of the cutting force on the force behavior of high-speed clamping chucks.

\section{FORCE ANALYSIS OF HIGH-SPEED CLAMPING CHUCKS}

When new devices are designed, the calculation of clamping forces can be reduced to solving the static problem on the workpiece equilibrium under the action of the external force, applied to it (Kuznetsov et al.,1977; Kuznetsov et al.,1988; Orlikov and Kuznetsov,.1977). All external forces are conditionally divided into disturbing and balancing workpiece (Fig. 1). Perturbing forces lead to the displacement of the workpiece from the position established at its basing. These include the components of the cutting force; volumetric forces (workpiece weight, centrifugal forces and inertia forces). The forces balancing the workpiece include frictional forces that arise when the workpiece is fixed; the force of direct action of power mechanisms on the workpiece; the reaction of supports.

If the cutting forces are always taken into account when calculating the clamping forces, then the volume forces are only for certain processing conditions. In particular, the weight of the workpiece is valid and is taken into account when it is installed on vertical and inclined elements. Centrifugal forces arise and are taken into account in the processing when the center of gravity of the workpiece is displaced relative to its axis of rotation.

Forces of inertia arise and are essential in two cases: when the workpiece performs reciprocating translation motion; at a sudden change in the speed rotational speed. The volume forces are determined from the relations given below.

Workpiece weight 
Citation: FW El-Dahabi, et al., Relations Power Characteristics of High-Speed Chucks with Power Cuts. Australian Journal of Basic and Applied Sciences, 13(7): 35-41. DOI: 10.22587/ajbas.2019.13.7.6

$G=\mathrm{mg}$,

where $m$ is the mass of the workpiece; $g$ - acceleration of gravity.

Centrifugal force

$\mathrm{F}_{\mathrm{c}}=\operatorname{mr}(\pi \mathrm{n} / 30)^{2}$

where is the rotational speed of the workpiece; $n r$ is the distance between the axis of rotation and the mass center of the workpiece.

The force of inertia, $F_{i}=$ ma,

Where $\mathrm{a}$ is the acceleration of the workpiece.

The frictional force is the main force factor that ensures the workpiece immobility from the action of disturbing external forces. It arises as a result of the action of the fixation force and is determined from the relation $\mathrm{F}_{\mathrm{f}}=$ Q.f,

Where $\mathrm{Q}$ is the fixing force; $\mathrm{f}$ is the coefficient of friction.

Perturbing forces

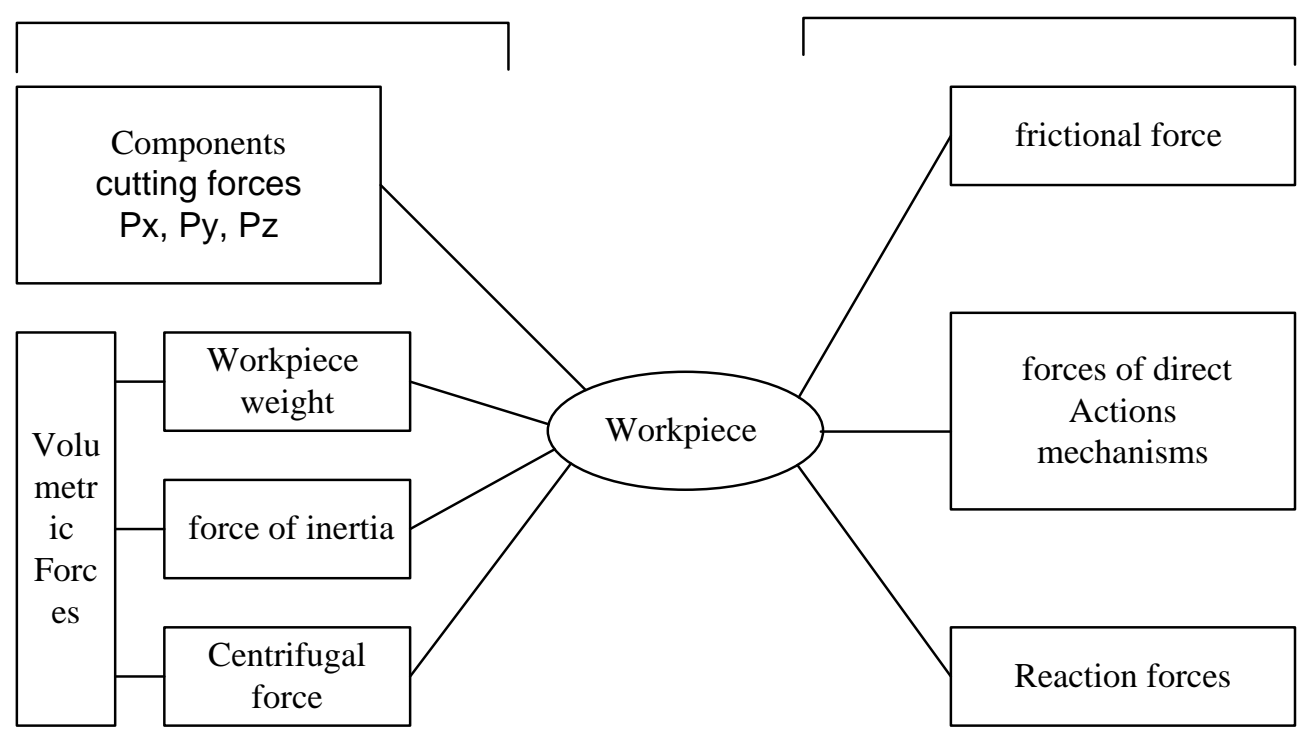

Figure.1: Forces acting on the workpiece

To calculate the binding forces, it has to exist:

1 - Working drawing of the workpiece for the operation (operations) performed, indicating the surface to be treated (surfaces);

2 - Detailed drawing of the part to be obtained when machining using the tool;

3 - Methods of processing the given surface (surfaces) of the workpiece and the necessary equipment and tools;

4 - Cutting modes (cutting speed, depth of cut and feed) and cutting force components;

5 - Workbench basing schemes and machine tooling system;

6 - Values of friction coefficients for various conditions of contact of workpiece surfaces with adjusting and clamping elements of devices;

7 - Values of safety factors for various conditions of fastening and processing of blanks.

To determine (in general form) the binding force when turning an external cylindrical surface with a passing cutter (Kovaleva N.V., Natalyutkina I.A.,2007), let us consider the scheme of forces acting on the workpiece being processed (Fig. 2). Let us consider the action of disturbing external forces. The circumferential (tangential) component force Pz has the greatest absolute value at turning and acts along the tangent to the workpiece. It creates a moment that tends to rotate the workpiece around its axis. The radial component force $\mathrm{P}_{\mathrm{y}}$, depending on the processing conditions, is $(0.3 \ldots 0.9) \mathrm{P}_{\mathrm{z}}$ and acts normal to the machined surface from the periphery to the center of the workpiece. It is opposed by the reaction from the cams. Since the radial force does not cause displacement of the workpiece, then, usually, the reaction scheme is not shown. The axial component force Px, depending on the processing conditions, is $(0.1 \ldots 0.7) \mathrm{Pz}$ and acts on the workpiece in the direction of tool delivery. At the same time, it tries 
to move the workpiece along the axis, the workpiece is fixed by the force of fastening $Q$, applied to the workpiece from the chucks of the cartridge.

In this case, the value of the fixing force must be such that the possibility of turning the workpiece in the cartridge from the action of the circumferential force, its displacement along the axis caused by the axial force, is excluded. The possibility of rotating the workpiece around the Ox axis in the cartridge and its displacement along the Ox axis is eliminated by the action of friction forces $F_{1}$ and $F_{2}$. They arise as a result of the action of the binding force at the point of contact between the cams of the cartridge and the base surface of the workpiece. And it is part $\mathrm{Q}_{1}$ counteracts the rotation of the workpiece around its axis, and $\mathrm{Q}_{2}$ counteracts the displacement of the workpiece along the axis. Then, the fixing force will be

$Q=k \sqrt{Q_{1}^{2}+Q_{2}^{2}}$

where $\mathrm{Q}_{1}$ is the part of the clamping force counteracting the rotation of the workpiece around the axis; $\mathrm{Q}_{2}-$ part of the clamping force counteracting the displacement of the workpiece along the axis; $\mathrm{k}$ - the safety factor depends on the specific processing conditions.

To ensure the immobility of the workpiece under the action of all external forces, we will compose the equations of static equilibrium

$\sum M_{o x}=n F_{T p 2} \frac{D_{2}}{2}-P_{Z} \frac{D_{1}}{2}=0$
$\sum P_{o x}=n F_{T p 1}-P_{X}=0$

Where $\mathrm{n}$ is the number of cams of the cartridge, for the three-jaw chuck $n=3$; is the diameter of the surface to be treated; $\mathrm{D}_{2}$ is the diameter of the reference surface; $P_{x}$ is the axial component of the cutting force; $P_{z}$ is the tangential component of the cutting force.
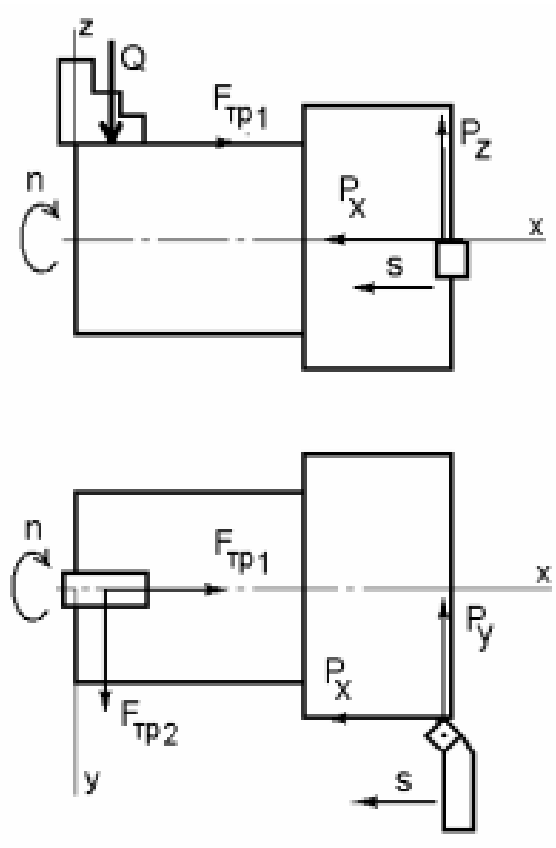

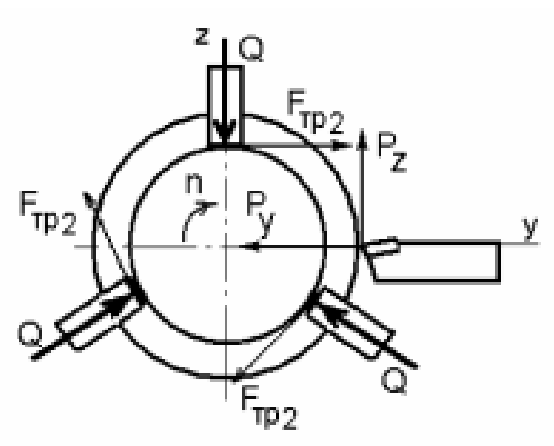

Figure.2: Calculation scheme for determining the fastening force for turning

Table 1: Cutting forces components

\begin{tabular}{|c|c|c|c|c|c|c|c|c|c|}
\hline \multirow{3}{*}{ Components of cutting force } & \multirow{3}{*}{ Tool material } & \multicolumn{8}{|c|}{ Processed metal } \\
\cline { 3 - 10 } & & \multicolumn{3}{|c|}{ steel, aluminum, alloys } & \multicolumn{4}{|c|}{ Cast iron, bronze } \\
\cline { 3 - 10 } & & $\mathrm{C}_{\mathrm{p}}$ & $\mathrm{x}_{\mathrm{p}}$ & $\mathrm{y}_{\mathrm{p}}$ & $\mathrm{z}_{\mathrm{p}}$ & $\mathrm{C}_{\mathrm{p}}$ & $\mathrm{x}_{\mathrm{p}}$ & $\mathrm{y}_{\mathrm{p}}$ & $\mathrm{z}_{\mathrm{p}}$ \\
\hline \multirow{2}{*}{$\mathbf{P}_{\mathbf{x}}$} & Hard alloy & 0,21 & 1,2 & 0,65 & 1,5 & 0,51 & 1,2 & 0,65 & 1,1 \\
\hline \multirow{2}{*}{$\mathbf{P}_{\mathbf{y}}$} & High speed steel & 0,21 & 1,2 & 0,65 & 1,5 & 1,6 & 1,2 & 0,65 & 1,1 \\
\hline \multirow{2}{*}{$\mathbf{P}_{\mathbf{z}}$} & Hard alloy & 0,027 & 0,9 & 0,75 & 2 & 0,45 & 0,9 & 0,75 & 1,1 \\
\cline { 2 - 10 } & High speed steel & 0,027 & 0,9 & 0,75 & 2 & 1,3 & 0,9 & 0,75 & 1,3 \\
\hline & Hard alloy & 35,7 & 1 & 0,75 & 0,75 & 51,4 & 1 & 0,75 & 0,55 \\
\cline { 2 - 10 } & High speed steel & 27,9 & 1 & 0,75 & 0,35 & 63,5 & 1 & 0,75 & 0,55 \\
\hline
\end{tabular}


As can be seen, in all two equations, the action of all external disturbing forces (components of the cutting force) is completely compensated. From the volume forces only the weight of the workpiece acts, which has incomparably less value than other external forces.

Substituting the value of the frictional force into Eqs. (6) and (7),

$F_{f 1}=f Q_{1} \quad F_{f 2}=f Q_{2}$

We obtain

$\sum M_{o x}=3 f Q_{2} \frac{D_{2}}{2}-P_{Z} \frac{D_{1}}{2}=0$

$\sum P_{o x}=3 f Q_{1}-P_{X}=0$

From the equations (8) and (9) we find:

$Q_{1}=\frac{P_{X}}{3 f}$

$Q_{2}=\frac{P_{Z} D_{1}}{3 f D_{2}}$

After the transformations, we obtain the formula for calculating the binding force:

$Q=k \sqrt{\left(\frac{P_{X}}{3 f}\right)^{2}+\left(\frac{P_{Z} D_{1}}{3 f D_{2}}\right)^{2}}$

Components of the cutting forces

$\mathrm{P}_{\mathrm{x}}=\mathrm{C}_{\mathrm{p}} \cdot \mathrm{t}^{\mathrm{xp}} \cdot \mathrm{S}^{\mathrm{xp}} \cdot \mathrm{HB}^{\mathrm{xp}}$;

$\mathrm{P}_{\mathrm{y}}=\mathrm{C}_{\mathrm{p}} \cdot \mathrm{t}^{\mathrm{yp}} \cdot \mathrm{S}^{\mathrm{yp}} \cdot \mathrm{HB}^{\mathrm{yp}}$;

$\mathrm{P}_{\mathrm{z}}=\mathrm{C}_{\mathrm{p}} \cdot \mathrm{t}^{\mathrm{zp}} \cdot \mathrm{S}^{\mathrm{zp}} \cdot \mathrm{HB}^{\mathrm{zp}}$.

where $S$ is the feed (mm / tr); t- the depth of cutting (mm); HB- hardness; $C_{p}$-values of the coefficient; $X P, y p, z p$ - exponents of the degree

The values of coefficient $C_{p}$ and exponents of the powers of $x p, y p, z p$ in the equations of cutting force components are given in Table 1. We will perform a theoretical analysis of the power characteristics of the HSCC (a patent of Ukraine for invention No. 15246 "Lathe chuck") under various conditions [11] with hydraulic clamping drive.

\section{Clamping with the spindle stopped $(n=0)$, fig. 3}

The gain of the cartridge is $k_{k}=\frac{Q}{S}$;

There $Q$ is the radial clamping force of the workpiece developed by one cam, $S$ is the axial force of the actuator per cam and is equal to $S=p \cdot F_{\Gamma} \cdot \eta$ ( $p$ - liquid pressure in the hydraulic cylinder, $F_{h}$ - useful area of the hydraulic cylinder,

$\eta=0.95$ - efficiency hydraulic drive, taking into account the leakage and friction in the seals).

\section{Clamping with rotating spindle $(n \neq 0)$, Fig. 4}

The centrifugal force of the unbalanced cam of the cartridge

$$
F_{\ldots}=m_{K} \cdot R \cdot \omega^{2}=m_{K} \cdot R\left(\frac{\pi n}{30}\right)^{2}=0.11 m_{K} \cdot R \cdot n^{2}
$$

The CFI is

$$
k n=\frac{Q-F_{u}}{S}
$$




\section{Clamping with the rotating spindle $(n \neq 0)$ and balancing the centrifugal forces (Fig. 5$)$.}

The introduction of a counterweight - a load on the shoulder p from the opposite side of the cam leads to an increase in the coefficient of amplification of the lever transmission to the great $\kappa_{\mathrm{p}}^{\prime}=\frac{R_{2}}{R_{1}^{\prime}}$

Where $R_{1}$ - the force on the arm a of the lever, which becomes smaller due to the force of the $F_{L \Gamma}$ when receiving the same force $\mathrm{R}_{2}$. It follows from (12): $\mathrm{R}_{2}=\mathrm{R}_{1} \cdot \mathrm{k}_{\mathrm{P}}$

$F_{L \Gamma}=m_{\Gamma} \cdot R \cdot \omega^{2}=m_{\Gamma} \cdot R\left(\frac{\pi n}{30}\right)^{2}=0.11 m_{\Gamma} \cdot R \cdot n^{2}$

The CFI is

$$
k n=\frac{Q-F_{L K}+F_{Ц \Gamma}}{S}
$$

\section{CONCLUSION}

The above considerations, design schemes, and analytical dependencies can be used to design high-speed clamping chucks under given cutting conditions.

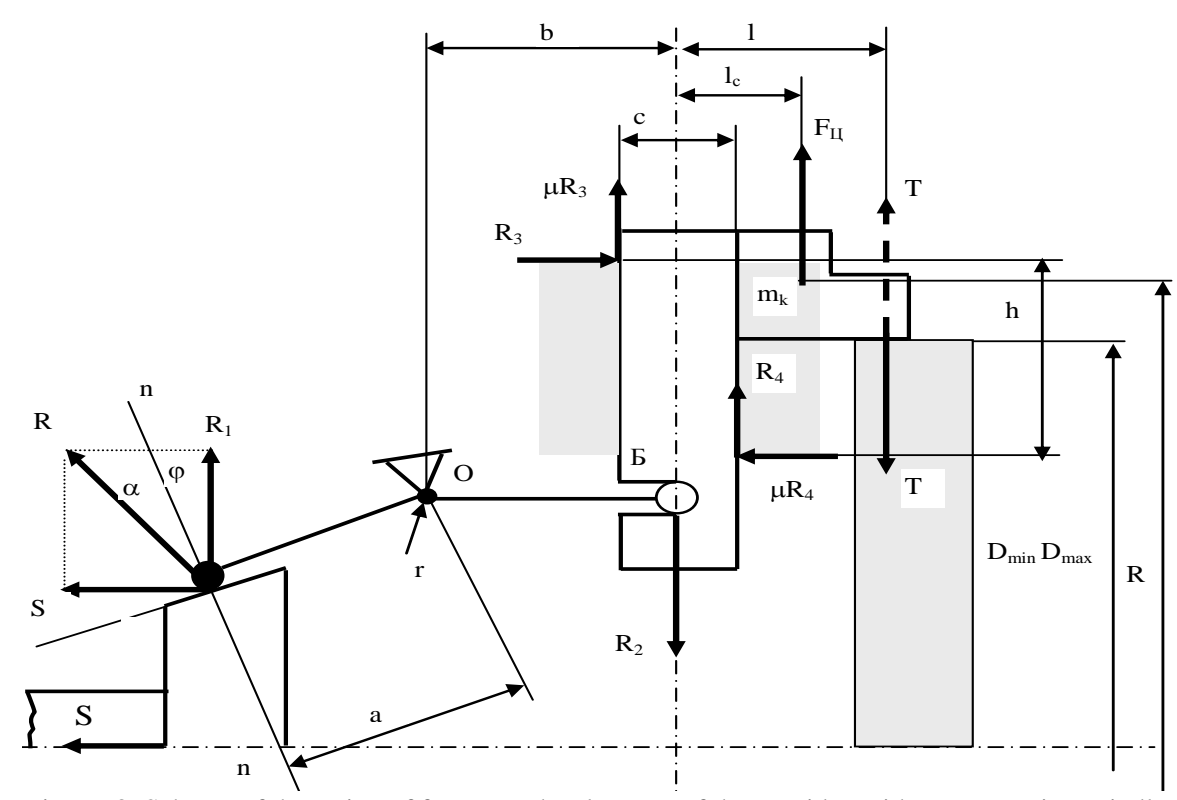

Figure. 3: Scheme of the action of forces on the elements of the cartridge with a non rotating spindle 


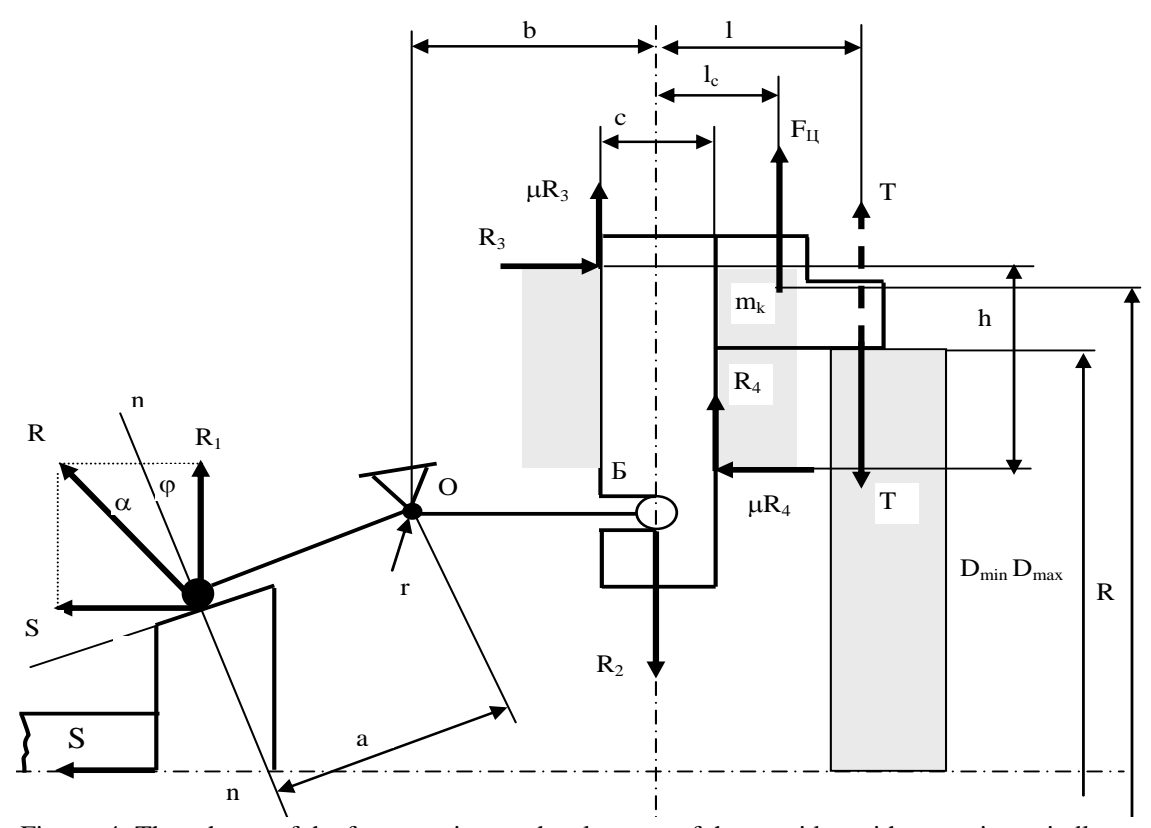

Figure. 4: The scheme of the forces acting on the elements of the cartridge with a rotating spindle

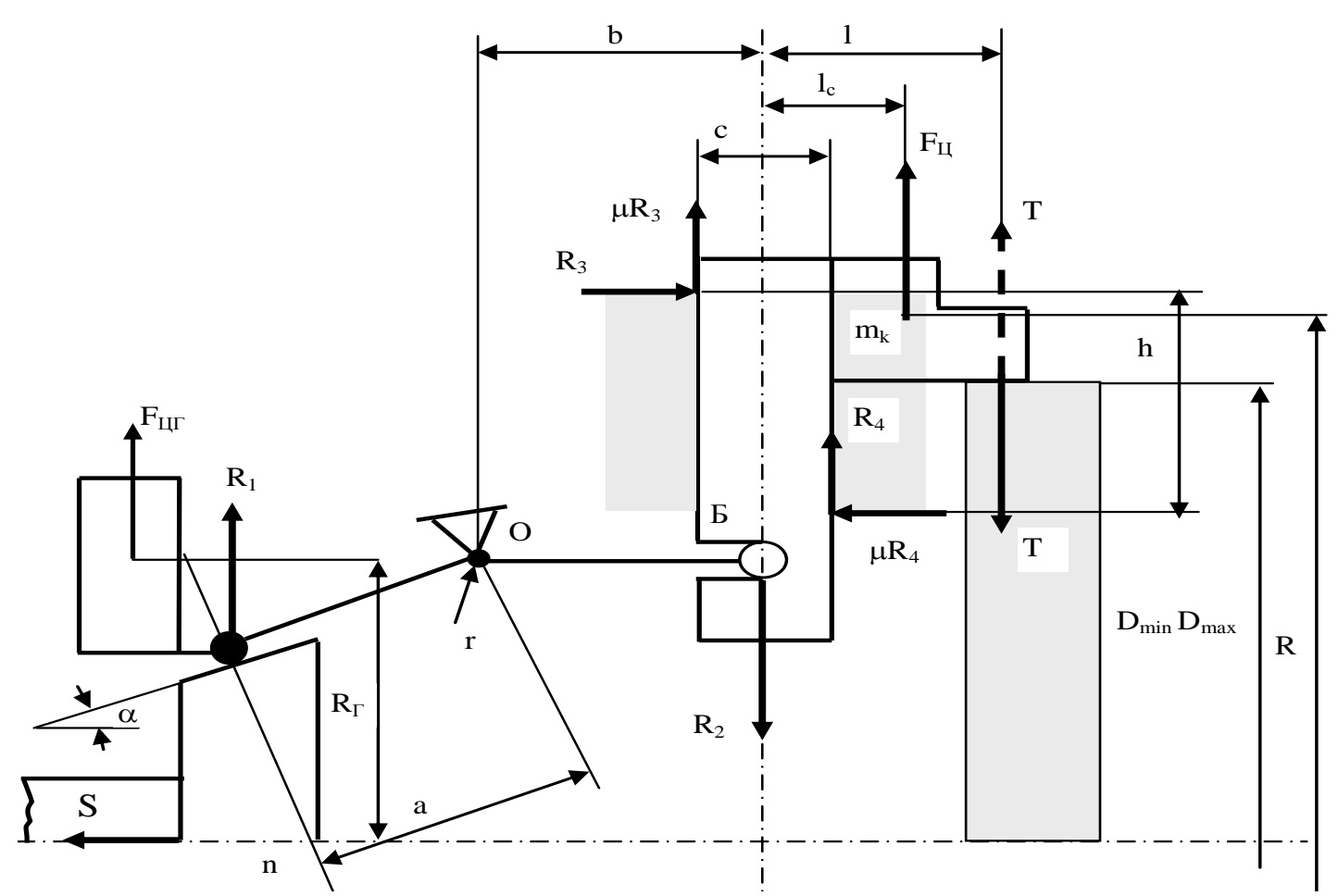

Figure. 5: The scheme of the forces acting on the elements of the cartridge with a rotating spindle and balancing the centrifugal forces

\section{REFERENCES}

Kuznetsov Y., Voloshin V., Nedelcheva P., El-Dahabi F.W., Clamping mechanisms for high-performance and high- precision machining, Monograph, Gabrovo, 2010

El-Dahabi F.W., Kuznetsov Y., Hazim M., Principles of designing high-speed clamping chucks, Bulletin of NTUU, Part: Machine Building, №72, 2014, 175-178

Al - Quraan Tareq. High-speed clamping mechanism of CNC lathe with compensation of centrifugal forces, Tareq Al- Quraan, Yuriy Kuznetsov, Taras Tsvyd., Procedia Engineering, vol.150, 2016. - pp. 689-695.

Al - Quraan Tareq. The effect of friction and joints rigidity on the power characteristics of rotating chucks, Tareq Al- Quraan, Yuriy Kuznetsov, Journal of Technical University of Gabrovo, vol.52, 2016., pp. 3-7. 
Gunter Spur. Handbuch der Fertigungstechnik, Gunter Spur, Theodor Stoferle, Carl Hanser Verlag Munchen Wien, Band 3/1 Spanen, 1979-614 p.

Wissig K.H., Schneider j. Probleme der Spannsicherheit beim Hochgewirdikeitsdrehen (Workpiece clamping of high-speed turning) VDI-Zeitcshrift.-199, N9, s.102-105.

Kuznetsov Y.N., Target mechanisms for automatic machines and CNC machines, Textbook, Part 3, ZMOK, Ternopol, 2001,354

Orlikov M.L., Kuznetsov Y.N., Designing clamping mechanisms of automated machine tools, Monograph, Machine Building, 1977,142

Kuznetsov Y.N., Vachev A.A., Syarov S.P., Carvenikov A.C., Self-adjusting clamping mechanisms, Handbook, Sofia, Tehnika, 1988,222

Kovaleva N.V., Natalyutkina I.A., Power calculation and mechanics of machine tools, Drujkovka, 2007, 60

Kuznetsov Y.N., El-Dahabi F.W., Study of the power characteristics of the chuck, operating at high frequencies of rotation, Bulletin of NTUU, Part: Machine Building, 2014, 45, 76 